\title{
Effect of duration of irrigation on the infectivity and reproduction of the root-knot and the reniform nematodes on some vegetable crops grown in two different soil types.
}

\author{
Ahmed, A. Farahat, Alsayed A. Alsayed and Shaimaa, F. Diab \\ Department of Zoology and Agric. Nematol., Fac. of Agric., Cairo University
}

\begin{abstract}
Influence of changes of soil moisture due to the durations of irrigation on the development and reproduction of the root-knot ( $M$. incognita) and the reniform ( $R$. reniformis) nematodes infecting eggplant, squash and cowpea in two different soil types was studied under greenhouse conditions. Results showed that the highest counts of the root-knot nematode were recorded on roots of eggplant grown in loamy sand soil and irrigated at the fourth day. On squash, the highest nematode counts were recorded on plants grown in sandy loam soil and irrigated every day, and on cowpea roots grown in the same soil type but irrigated every four days. Higher reproductivity values of the reniform nematode on eggplant roots were accomplished in loamy sand soil and irrigated every four days. Significant higher final population and rate of reproduction on squash and cowpea were recorded on plants grown in the same soil type but irrigated daily or every other day, respectively. The influence of the duration of irrigation on nematode development depend on many factors most of which are the soil type, duration of irrigation, nematode species as well as the growing crop. The prolonging irrigation period (decreasing soil moisture) significantly reduced the growth of the infected plants. Such effect was pronounced with the longest period between irrigation (3 days) in loamy sand soil.
\end{abstract}

Key words: $M$. incognita, $R$. reniformis, vegetables, soil type, soil moisture.

\section{Introduction}

Soil moisture varies considerably as a result of many biological and physical actions. In the broad sense, some of the most obvious factors regulating soil moisture are intensity and frequency of irrigation, rainfall, the drainage pattern, and the precipitation/evaporation ratio. Other factors governing the amount of moisture in nematode's environment include the amount of available soil moisture for the particular soil type, the stage of plant maturity, the length of growing seasons, the water requirements of individual plants or crops, and elements relating to individual characteristics of the leaves and roots. The relative capacity of moisture is so dependent upon the soil texture and other factors that determine the soil phase that they cannot be separated. The coarser the soil, the larger the pores; the smaller the total soil surface area, the less water retained (Norton, 1978). 
It has been shown that movement and the invasion of host roots by the root knot nematode Meloidogyne spp. and the reniform nematode, Rotylenchulus reniformis is reduced at low moisture (Couch and Bloom, 1960; Rebois, 1973). Daulton and Nusbaum (1962) studied the effect of soil moisture and relative humidity on root-knot nematode Meloidogyne javanica. They found a greater and more rapid reduction in egg viability in wet soil $(20.4 \%$ moisture) than in dry soil (3.4\% moisture). Eggs of the Southern Rhodesia population withstood exposure to both wet and dry soil better than those of Georgia population. The more rapid reduction in viability in wet soil may be due to relatively quick hatching of eggs and increased activity of larvae in this environment, resulting in depletion of stored energy. The tolerance differences, they added, of the $M$. javanica populations to extremes of soil moisture may be due to the different environmental conditions in the areas from which they were taken.

Because of the difficulty of maintaining constant soil moisture, little direct evidence can be gained on moisture and nematode increase. Kable and Mai (1968) found that the population increase of Pratylenchus penetrans was greatest at moderate soil moisture tensions ( $\mathrm{pF} 2$ to 3 ) but there was an interaction with soil moisture and soil type; the greater the amount of silt and clay present, the greater the soil moisture tension for plant growth. Khanizadeh et al. (1994) tested the relative susceptibility of five strawberry (Fragaria $x$ ananassa Duch.) cultivars to the root-knot nematode (Meloidogyne hapla) under three different soil water deficit levels and three nematode densities. Percent water deficit had a positive linear effect on the number of galls on strawberry roots, and on egg production and number of $M$. hapla larvae in the soil, regardless of cultivar type. In 2007, Bakonyi et al. stated that alteration of the nematode community structure induced by global change may have a considerable influence on the ecosystem functioning, however, it is not clear whether minor changes in soil temperature and/or moisture have any significant effect on nematode community structure. They modified soil temperature and moisture to the extent expected for near future due to global changes. They proved that community diversity and multivariate structure of the nematode community were sensitive to minute changes in soil temperature and moisture than different indices such as specific richness, maturity index, plant parasite index. Nematode genera with high densities ( $>0.1$ individual g- 1 soil) were better indicators of the temperature and moisture changes than those of low density $(<0.1$ individual $\mathrm{g}-1$ soil) in sandy soil.

This study was undertaken to assess the influence of soil moisture and soil types as abiotic factors on the development and reproduction of the root-knot and reniform nematodes on some vegetable crops. 


\section{Materials and methods}

Pure cultures of the root-knot nematode, Meloidogyne incognita and the reniform nematode, Rotylenchulus reniformis obtained from the stock cultures belong to Nematology Research Center, Department of Zoology and Agric. Nematology, Faculty of Agriculture. Eggplant, Solanum melongena (gloyana F1 hyb.), squash, Cucurbita maxima (Tabarak F1 hyb.) and cowpea, Vigna unguiculata (cv Qaha 1) were used in the present study.

Influence of the changes of soil moisture due to the durations of irrigation on the development and reproduction of the root-knot and the reniform nematodes infecting eggplant, squash and cowpea was studied under the greenhouse conditions of $30 \pm 5^{\circ} \mathrm{C}$. The experiment was conducted in two soil types loamy sand and sandy loam in $15 \mathrm{~cm}$ diameter clay pots (Table 1). Soil types were mechanically analysed at Reclamation \& development Center for Desert Soils, Soil Department, Fac. of Agric. Cairo University. After cultivation, plants (one/pot) were inoculated with 2000 infective stages of either $M$. incognita or $R$. reniformis. Irrigation durations were at $1,2,3$ and 4 days intervals with $200 \mathrm{ml}$ of tab water throughout the experimental time (6 weeks). Four replicates were used for each irrigation time. Pots of each nematode species were arranged randomly on a clean bench of the greenhouse. At the end of experiment plants were taken off, plant growth criteria were measured and soil and root nematode populations were counted.

The obtained results were statistically analyzed according to SPSS software package version 12 (SPSS, 2003).

Table (1): Mechanical analysis of soil.

\begin{tabular}{lccccc}
\hline \multirow{2}{*}{ Soil type } & \multicolumn{5}{c}{$\%$ Soil particles } \\
\cline { 2 - 6 } & Coarse sand & Fine sand & Silt & Clay & Texture class \\
\hline $\mathbf{1}$ loam :1 sand $(\mathbf{v}: \mathbf{v})$ & 47.7 & 33.6 & 9.1 & 8.9 & Loamy sand \\
Loamy soil & 1.8 & 70.6 & 11.3 & 16.3 & Sandy loam \\
\hline
\end{tabular}

Results

\section{A. The root-knot nematode}

Data present in table (2) indicate that the highest significant number of galls formed on eggplant roots was accomplished on plants grown in loamy sand soil and irrigated at the fourth day (the longest period that plants tolerate water deficiency) followed by those grown in sandy loam soil and irrigated day after day. The highest numbers of egg-masses were also formed in the same treatment which varied significantly with the other treatments in both types of soil. Concerning the number 
Table (2): Impact of irrigation duration on the development and reproduction of $M$. incognita infecting vegetable arops grown in two types of soil.

\begin{tabular}{|c|c|c|c|c|c|c|c|c|c|c|}
\hline Crop & soll ty pe & $\begin{array}{l}\text { Irri gation } \\
\text { duration }\end{array}$ & Galls/root & $\begin{array}{c}\text { Eggma } 65 \text { es/ } \\
\text { Root }\end{array}$ & $\begin{array}{c}\text { Eggsl } \\
\text { eggmass }\end{array}$ & $\begin{array}{c}\text { Total no. } \\
\text { eggs }\end{array}$ & Eggs/g soll & $\begin{array}{c}\text { Soll } \\
\text { population }\end{array}$ & $\begin{array}{c}\text { Final } \\
\text { population }\end{array}$ & $\mathrm{PTPI}$ \\
\hline \multirow{8}{*}{$\begin{array}{l}\text { Eggpla nt } \\
\text { Gloy ana F1 }\end{array}$} & Loamy sand & Every day & 312.8 of & $187.0 \mathrm{DC}$ & $249.8 \mathrm{C}$ & $46712.6 \mathrm{C}$ & $23.4 \mathrm{C}$ & 0.0 & $187.0 \mathrm{bc}$ & $0.09 \mathrm{bc}$ \\
\hline & & $2^{\text {nd }} \mathrm{day}$ & 293.80 & $207.8 \mathrm{bC}$ & $323.8 \mathrm{D}$ & $67285.6 \mathrm{~b}$ & $33.6 \mathrm{~b}$ & 0.0 & $207.8 \mathrm{bc}$ & $0.10 \mathrm{bc}$ \\
\hline & & $3^{\text {nd }} \mathrm{day}$ & $307.0 \mathrm{cot}$ & $225.8 \mathrm{~b}$ & $336.3 \mathrm{~b}$ & $75936.5 \mathrm{~b}$ & $380 \mathrm{D}$ & 0.0 & $225.8 \mathrm{~b}$ & 0.110 \\
\hline & & $4^{\text {th }}$ day & $501.3 \mathrm{a}$ & $3140 \mathrm{a}$ & 557.3 a & $1749922 \mathrm{a}$ & $87.5 \mathrm{a}$ & $1200 \mathrm{a}$ & $434.0 \mathrm{a}$ & $0.22 \mathrm{a}$ \\
\hline & Sandy loam & Every day & $380.0 \mathrm{~b}$ & $182.5 \mathrm{DC}$ & 173.80 & $31718.5 d$ & $16.0 \mathrm{~d}$ & 0.0 & $182.5 \mathrm{bc}$ & $0.09 \mathrm{bc}$ \\
\hline & & $z^{\text {nd }} d a y$ & $392.0 \mathrm{~b}$ & $200.3 \mathrm{bc}$ & $223.0 \mathrm{C}$ & $44666.9 \mathrm{C}$ & $224 \mathrm{C}$ & 0.0 & $200.3 \mathrm{bc}$ & $0.10 \mathrm{bc}$ \\
\hline & & $3^{n d} d a y$ & $354.0 \mathrm{bc}$ & $175.3 \mathrm{c}$ & $1170 \mathrm{e}$ & $20510.1 \mathrm{e}$ & $10.3 \mathrm{e}$ & 0.0 & $175.3 \mathrm{c}$ & $0.09 \mathrm{c}$ \\
\hline & & $4^{\text {th }}$ day & $293.3 \mathrm{~d}$ & $2020 \mathrm{bc}$ & 18000 & 36360.000 & 18200 & 0.0 & $202.0 \mathrm{bc}$ & $0.10 \mathrm{bc}$ \\
\hline \multirow{8}{*}{$\begin{array}{l}\text { Squash } \\
\text { Tabarak F1 }\end{array}$} & Loamy sand & Every day & 716.5 a & $120.8 \mathrm{DC}$ & $9.5 \mathrm{c}$ & $1147.6 \mathrm{C}$ & $0.6 \mathrm{c}$ & 0.0 & $120.8 b c$ & $0.05 \mathrm{bc}$ \\
\hline & & $2^{n d} \mathrm{dgy}$ & $578.0 \mathrm{O}$ & $154.3 \mathrm{~b}$ & $12.3 \mathrm{c}$ & $13979 \mathrm{C}$ & $1.0 \mathrm{c}$ & 0.0 & $154.3 \mathrm{~b}$ & 0.080 \\
\hline & & $3^{\text {nd }} d x y$ & $4545 \mathrm{C}$ & 108.800 & $16.0 \mathrm{c}$ & $1740.8 \mathrm{C}$ & $09 \mathrm{c}$ & 0.0 & 108.8 od & 0.05 od \\
\hline & & $4^{\text {th }}$ day & 316.50 & 87.3 od & $160 \mathrm{c}$ & $1396.8 \mathrm{C}$ & $0.7 \mathrm{c}$ & 0.0 & 87.3 od & 0.040 \\
\hline & Sandy loam & Every day & $564.5 \mathrm{~b}$ & $256.8 \mathrm{a}$ & 291.3 a & $74805.8 \mathrm{a}$ & $37.4 \mathrm{a}$ & 0.0 & $256.8 \mathrm{a}$ & $0.13 \mathrm{a}$ \\
\hline & & $2^{\text {nd }} \mathrm{dgy}$ & $4348 \mathrm{C}$ & $113.8 \mathrm{c}$ & 17200 & $19573.6 \mathrm{bc}$ & $9.8 \mathrm{bc}$ & $1340 \mathrm{D}$ & $247.8 \mathrm{a}$ & $0.12 \mathrm{a}$ \\
\hline & & $3^{\text {ed }} \mathrm{day}$ & 281.80 & $122.8 \mathrm{DC}$ & $237.3 \mathrm{ab}$ & $29140.4 \mathrm{~b}$ & $14.6 \mathrm{~b}$ & $1620 \mathrm{a}$ & $284.8 \mathrm{a}$ & $0.14 \mathrm{a}$ \\
\hline & & $4^{\text {th }}$ day & $170.0 \mathrm{e}$ & 70.80 & $239.8 \mathrm{ab}$ & $16977.8 \mathrm{bc}$ & $8.5 \mathrm{bc}$ & 0.0 & 70.80 & 0.040 \\
\hline \multirow{8}{*}{$\begin{array}{l}\text { Cowpea } \\
\text { Caha } 1\end{array}$} & Loamy sand & Every day & 491.3 of & $351.8 \mathrm{c}$ & $4500 \mathrm{e}$ & $158310.0 \mathrm{~cd}$ & 792 of & 12448 od & 1596.5 of & 0.20 os \\
\hline & & $2^{p d} d a y$ & 396.80 & 260.80 & $777.8 \mathrm{ab}$ & $2028502 \mathrm{C}$ & $101.4 \mathrm{c}$ & 813.30 & 107400 & 0.540 \\
\hline & & $3^{n d} \mathrm{day}$ & $530.0 \mathrm{bc}$ & $375.0 \mathrm{C}$ & 568.30 & $213112.5 \mathrm{C}$ & $105.6 \mathrm{c}$ & $3776.0 \mathrm{~b}$ & $4151.0 \mathrm{~b}$ & $2.08 \mathrm{~b}$ \\
\hline & & $4^{\text {th }}$ day & $511.3 \mathrm{dr}$ & $375.3 \mathrm{c}$ & 8060 a & $302491.8 \mathrm{D}$ & $151.2 \mathrm{~b}$ & $1968.3 \mathrm{C}$ & $2343.5 \mathrm{c}$ & $1.17 \mathrm{c}$ \\
\hline & Sandy loam & Every day & $650.8 \mathrm{~b}$ & $439.8 \mathrm{~b}$ & $469.3 \mathrm{e}$ & $229853.1 \mathrm{C}$ & $114.9 \mathrm{c}$ & 1007.5 od & 1497.3 od & 0.75 od \\
\hline & & $2^{\text {nd }} \mathrm{day}$ & $532.8 \mathrm{bc}$ & $173.3 \mathrm{e}$ & $661.8 \mathrm{C}$ & 1146899 व & 57.30 & 413.30 & 586.50 & 0.290 \\
\hline & & $3^{n d} d a y$ & $636.5 \mathrm{~b}$ & 316.0 or & $354.8 \%$ & 112116.80 & 56.10 & $1316.0 \mathrm{dr}$ & $1632.0 \mathrm{or}$ & 0.82 od \\
\hline & & $4^{\text {th }}$ day & $832.8 \mathrm{a}$ & $721.3 \mathrm{a}$ & $734.5 \mathrm{D}$ & 5297949 a & $264.9 \mathrm{a}$ & $6534.0 \mathrm{a}$ & $7255.3 \mathrm{a}$ & $3.63 \mathrm{a}$ \\
\hline
\end{tabular}

In each column, vaues of a crop cu rivars to lowed by the same etwer(s) are not signilicantly d fterent $(P=0.05)$. 
of eggs/egg-mass, the number of eggs/g soil as well as the total number of eggs/root, the highest numbers were formed on plants grown in loamy sand soil and irrigated at 4, 3, 2 days intervals, in that order. Significant lower numbers of such counts were observed on roots of plants grown in sandy loam soil. As well, the final population and the rate of nematode build up was recorded in loamy sand soil irrigated every four days.

On squash, the highest significant numbers of egg-masses/root, eggs/eggmass, eggs/g soil, total number of eggs/plant, final population and rate of nematode build up were recorded on plants grown in sandy loam soil and irrigated every day. Except for number of galls and egg-masses /root, other nematode counts are higher in sandy loam soil than those in loamy sand soil and irrigated at different intervals. On cowpea, different behavior was observed whereas the highest significant nematode counts were recorded on roots of plants grown in sandy loam soil and irrigated every four days followed by those grown in loamy sand and irrigated at the same interval. Apparently, no significant differences could be observed within and between different irrigation intervals in both types of soil.

Concerning growth response of the tested vegetables, data in table (3) indicated that growth criteria (especially shoot criteria) of plants grown in loamy sand either infected or none infected with the root-knot nematode are significantly higher than those grown in sandy loam soil. Increasing the intervals of irrigation adversely affected the growth of infected plants of the three vegetables in both types of soil. The longer the period between irrigation intervals the greater the reduction in plant growth.

\section{B. The reniform nematode}

Data in table (4) designated that the highest numbers of egg-masses/root, eggs/g soil, total number of eggs, final population as well as the rate of reproduction on eggplant roots were accomplished in loamy sand soil irrigated every four days. Irrespective of intervals of irrigation, nematode counts in the loamy sand soil were significantly higher than those in the sandy loam soil. Generally, increasing soil moisture (daily irrigation) was not in favor to nematode reproduction, however, prolonging the period of irrigation enhanced the reniform nematode reproduction. Conversely, increasing soil moisture (daily irrigation or irrigation each another day) was found to be in favor to nematode reproduction on squash and cowpea especially in loamy sand soil. The most significant highest final population and rate of reproduction on squash was recorded in loamy sand soil irrigated every day and on cowpea in sandy loam soil irrigated every other day.

Regarding growth response to the infection of the reniform nematode and the stress of soil moisture, data in table (5) illustrate that prolonging irrigation period (decreasing soil moisture) significantly reduced the growth of the infected plants. Such effect was pronounced with the longest period between irrigation (3 days) and in loamy sand soil. 
Table (3): Growth of eggplant, squash and cowpeg infected with $M$. incognita and grown in two different soil types as affected by the duration of irrigation.

\begin{tabular}{|c|c|c|c|c|c|c|c|c|c|}
\hline \multirow{2}{*}{ Crop } & \multirow{2}{*}{$\begin{array}{l}\text { soll } \\
\text { ty } p=\end{array}$} & \multirow{2}{*}{$\begin{array}{c}\text { Irrigation } \\
\text { duration }\end{array}$} & \multicolumn{3}{|c|}{ s noot } & \multicolumn{2}{|c|}{ Root } & \multirow{2}{*}{$\begin{array}{c}\begin{array}{c}\text { Total } \\
\text { length }\end{array} \\
\text { (cm) }\end{array}$} & \multirow{2}{*}{$\begin{array}{c}\begin{array}{c}\text { Total fres } \\
\text { wel ght }\end{array} \\
\text { (gm) }\end{array}$} \\
\hline & & & length $(\mathrm{cm})$ & fres $\mathrm{h}$ wel ght (gm) & dry w elght (gm) & langth (cm) & fresh welght (gm) & & \\
\hline \multirow{10}{*}{$\begin{array}{l}\text { Eggplant } \\
\text { Gloya na F1 }\end{array}$} & \multirow{5}{*}{$\begin{array}{l}\text { Loamy } \\
\text { sand }\end{array}$} & Every day & $26.88 \mathrm{a}$ & $4.68 \mathrm{a}$ & $0.56 \mathrm{~b}$ & 15.330 & 2.000 & $4220 \mathrm{bc}$ & $6.68 \mathrm{ab}$ \\
\hline & & $2^{\text {nd day }}$ & $26.13 \mathrm{a}$ & $4.63 \mathrm{a}$ & $0.62 \mathrm{~b}$ & $17.68 \mathrm{C}$ & $1.27 \mathrm{bc}$ & $43.80 \mathrm{D}$ & $5.90 \mathrm{~b}$ \\
\hline & & $3^{\text {dd }}$ day & $25.28 \mathrm{a}$ & $3.15 \mathrm{~b}$ & $0.45 \mathrm{c}$ & 14.07 de & 0.500 & $39.34 \mathrm{c}$ & $3.65 \mathrm{C}$ \\
\hline & & $4^{\text {th }}$ day & $22.75 \mathrm{D}$ & $2.93 \mathrm{bc}$ & $0.44 \mathrm{c}$ & 13.33 de & $0.85 \mathrm{dr}$ & $36.08 d$ & $3.78 \mathrm{c}$ \\
\hline & & Healthy & $26.50 \mathrm{a}$ & $4.60 \mathrm{a}$ & $0.95 \mathrm{a}$ & $26.00 \mathrm{a}$ & $2.91 \mathrm{a}$ & $52.50 \mathrm{a}$ & $7.51 \mathrm{a}$ \\
\hline & \multirow{5}{*}{$\begin{array}{l}\text { Sandy } \\
\text { loam }\end{array}$} & Every day & $20.50 \mathrm{c}$ & $1.40 \mathrm{e}$ & $0.16 \mathrm{e}$ & $14.00 \mathrm{de}$ & $1.48 \mathrm{bc}$ & $34.50 \mathrm{de}$ & $2.88 \mathrm{C}$ \\
\hline & & $2^{2 d}$ day & $19.13 \mathrm{c}$ & $1.57 \mathrm{e}$ & $0.18 \mathrm{e}$ & $14.88 \mathrm{de}$ & $1.40 \mathrm{dc}$ & $34.00 \mathrm{de}$ & $2.97 \mathrm{C}$ \\
\hline & & $y^{\text {dd }}$ day & $20.38 \mathrm{C}$ & 2.43 od & 0.270 & 13.63 de & $1.28 \mathrm{bc}$ & $34.00 \mathrm{de}$ & $3.71 \mathrm{C}$ \\
\hline & & $4^{\text {th }}$ day & $19.63 \mathrm{C}$ & $2.61 \mathrm{bc}$ & 0.24 de & $1288 \mathrm{e}$ & $1.35 \mathrm{bc}$ & $3250 \mathrm{e}$ & $3.96 \mathrm{c}$ \\
\hline & & Healthy & $20.50 \mathrm{C}$ & $1.79 \mathrm{de}$ & $0.55 \mathrm{~b}$ & $2000 \mathrm{~b}$ & $1.40 \mathrm{bc}$ & $40.50 \mathrm{c}$ & $3.19 \mathrm{c}$ \\
\hline \multirow{10}{*}{$\begin{array}{l}\text { Squash } \\
\text { Tabarak F1 }\end{array}$} & \multirow{5}{*}{$\begin{array}{l}\text { Loamy } \\
\text { sand }\end{array}$} & Every day & $32.05 a b$ & 4.880 & 0.490 & $18.68 \mathrm{C}$ & 1.050 & $50.73 \mathrm{~b}$ & $5.92 \mathrm{C}$ \\
\hline & & $2^{\text {ad }}$ day & 25.30 or & $2.80 \mathrm{c}$ & $0.27 \mathrm{e}$ & 16.680 & $1.05 \mathrm{~b}$ & $41.98 \mathrm{c}$ & 3.250 \\
\hline & & $3^{\text {td }}$ day & 25.35 or & $3.13 \mathrm{c}$ & $0.20 \mathrm{e}$ & $11.68 \mathrm{e}$ & $0.57 \mathrm{c}$ & 37.030 & 3.710 \\
\hline & & $4^{\text {th }}$ day & 23.000 & $2.70 \mathrm{c}$ & $0.26 \mathrm{e}$ & $1268 \mathrm{e}$ & $0.50 \mathrm{c}$ & $35.68 d$ & $3.20 \mathrm{de}$ \\
\hline & & Heaithy & $33.05 \mathrm{a}$ & $7.70 \mathrm{a}$ & $1.20 \mathrm{a}$ & $2400 \mathrm{D}$ & $2.45 \mathrm{a}$ & $57.05 \mathrm{a}$ & $10.15 \mathrm{a}$ \\
\hline & \multirow{5}{*}{$\begin{array}{l}\text { Sandy } \\
\text { loam }\end{array}$} & Every day & $28.70 \mathrm{bC}$ & $7.25 \mathrm{a}$ & $0.73 \mathrm{C}$ & $20.38 \mathrm{C}$ & $1.22 \mathrm{~b}$ & $49.08 \mathrm{D}$ & $8.47 \mathrm{~b}$ \\
\hline & & $2^{\text {nd }}$ day & 24.430 & $1.73 \mathrm{~d}$ & 0.35 de & $12.75 \mathrm{e}$ & $0.64 \mathrm{c}$ & 37.180 & $2.38 \mathrm{et}$ \\
\hline & & $3^{\text {rd }}$ day & 23.030 & $2.97 \mathrm{C}$ & $0.30 \mathrm{e}$ & $1300 \mathrm{e}$ & $0.61 \mathrm{c}$ & 36.030 & 3.580 \\
\hline & & $4^{\text {th }}$ day & $18.13 \mathrm{e}$ & 1.300 & $0.25 \mathrm{e}$ & $9.50 \mathrm{f}$ & $0.28 \mathrm{c}$ & $27.63 \mathrm{e}$ & $1.58 \mathrm{f}$ \\
\hline & & Healthy & $31.00 a b$ & $7.21 \mathrm{a}$ & $1.00 \mathrm{~b}$ & $26.50 \mathrm{a}$ & $2.40 \mathrm{a}$ & $57.50 \mathrm{a}$ & $9.61 \mathrm{a}$ \\
\hline
\end{tabular}


Table (3) Continued

\begin{tabular}{|c|c|c|c|c|c|c|c|c|c|}
\hline \multirow{2}{*}{ crop } & \multirow{2}{*}{$\begin{array}{l}\text { solll } \\
\text { type }\end{array}$} & \multirow{2}{*}{$\begin{array}{l}\text { Imrigat on } \\
\text { duration }\end{array}$} & \multicolumn{3}{|c|}{ shoot } & \multicolumn{2}{|c|}{ Root } & \multirow{2}{*}{$\begin{array}{c}\begin{array}{c}\text { Total } \\
\text { length }\end{array} \\
(\mathrm{cm})\end{array}$} & \multirow{2}{*}{$\begin{array}{c}\text { Total fresh } \\
\text { welght }\end{array}$} \\
\hline & & & length(cm) & tresh welght (gm) & dry w elght (gm) & length $(\mathrm{cm})$ & $\begin{array}{c}\text { fres h welght } \\
\text { (gm) }\end{array}$ & & \\
\hline \multirow{10}{*}{$\begin{array}{l}\text { Cowpea } \\
\text { Qaha } 1\end{array}$} & \multirow{5}{*}{$\begin{array}{l}\text { Loamy } \\
\text { sa nd }\end{array}$} & Every day & $25.50 \mathrm{~b}$ & $3.33 \mathrm{de}$ & 0.50 or & $23.00 \mathrm{~b}$ & 195 or & $48.50 \mathrm{~b}$ & 5.280 \\
\hline & & $2^{\text {nd day }}$ & $23.15 \mathrm{bc}$ & 3.10 de & 0.45 de & $18.00 \mathrm{c}$ & 1.51 ode & $41.15 \mathrm{c}$ & 4.61 de \\
\hline & & $3^{\text {rd }}$ day & 22.03 ose & $2.23 \mathrm{ef}$ & 0.31 ef & 16.08 od & $2.00 \mathrm{c}$ & 38.10 od & $4.23 \mathrm{e}$ \\
\hline & & $4^{\text {th }}$ day & 22.75 bod & $2.80 \mathrm{de}$ & 0.39 de & 14.330 & 1.36 def & 37.08 dr & $4.16 \mathrm{e}$ \\
\hline & & Heal thy & $33.00 \mathrm{a}$ & $11.20 \mathrm{a}$ & $1.40 \mathrm{a}$ & $27.18 \mathrm{a}$ & $4.20 \mathrm{a}$ & $60.18 \mathrm{a}$ & $15.40 \mathrm{a}$ \\
\hline & \multirow{5}{*}{$\begin{array}{l}\text { Sa ndy } \\
\text { loam }\end{array}$} & Every day & 22.13 cose & $4.50 \mathrm{c}$ & $0.65 \mathrm{c}$ & $18.38 \mathrm{C}$ & $296 \mathrm{~b}$ & $40.51 \mathrm{C}$ & $7.46 \mathrm{c}$ \\
\hline & & $2^{\text {td day }}$ & 20.13 de & 3.5300 & 0.4900 & $15.68 \mathrm{dr}$ & 1.40 odef & 35.800 & 4.93 de \\
\hline & & $3^{n d}$ day & $19.73 \mathrm{e}$ & $1.13 \mathrm{~g}$ & $0.21 \mathrm{f}$ & 14.000 & $0.79 \mathrm{f}$ & $33.73 \mathrm{de}$ & $192 \mathrm{f}$ \\
\hline & & $4^{\text {th }}$ day & $16.96 \mathrm{f}$ & $1.20 \% 9$ & $0.21 \%$ & $13.63 d$ & $1.10 \mathrm{ef}$ & 30.59 e & $2.30 i$ \\
\hline & & Heal thy & $23.00 \mathrm{bc}$ & $6.20 \mathrm{~b}$ & $1.07 \mathrm{~b}$ & $24.00 \mathrm{~b}$ & $390 \mathrm{a}$ & $47.00 \mathrm{D}$ & $10.70 \mathrm{~b}$ \\
\hline
\end{tabular}

in each column, values of a cop cu rivars fo lowed by the same letter $(8)$ are not significantly orferent $(\mathrm{P}=0.05)$. 
Table (4): Impact of irrigation duration on the development and reproduction of R. reniformis infecting vegetable in two types of soil.

\begin{tabular}{|c|c|c|c|c|c|c|c|c|c|c|c|}
\hline Crop & soll type & $\begin{array}{l}\text { Irrl gation } \\
\text { duration }\end{array}$ & D. stages & $\begin{array}{l}\text { Mature } \\
\text { females }\end{array}$ & $\begin{array}{l}\text { Eggmasses/ } \\
\text { roct }\end{array}$ & $\begin{array}{c}\text { Eggs/ } \\
\text { eggma } 56\end{array}$ & $\begin{array}{c}\text { Total no. } \\
\text { eggs }\end{array}$ & $\underset{5011 / 6}{E g g 5 / g}$ & $\begin{array}{c}\text { Soll } \\
\text { population }\end{array}$ & $\begin{array}{c}\text { Final } \\
\text { population }\end{array}$ & PIIPI \\
\hline \multirow{8}{*}{$\begin{array}{l}\text { Eggpiant } \\
\text { Gloyana F1 }\end{array}$} & Loamy & Every day & 2.300 & $7.8 \mathrm{de}$ & $126.3 \mathrm{de}$ & $95.3 \mathrm{a}$ & $12036.4 \mathrm{bc}$ & $6.0 \mathrm{bc}$ & $1222.3 \mathrm{a}$ & $1358.7 \mathrm{a}$ & $0.68 \mathrm{a}$ \\
\hline & sand & $2^{\text {nd }} \mathrm{day}$ & $5.3 \mathrm{a}$ & 900 & $166.3 \mathrm{c}$ & $20.8 \mathrm{~b}$ & 13437.00 & $6.7 \mathrm{~b}$ & 321.8 od & $5023 \mathrm{c}$ & $0.25 \mathrm{c}$ \\
\hline & & $3^{\text {nd }}$ day & 1.80 & 6.0 de & $203.0 \mathrm{~b}$ & 52.80 & $10718.4 \mathrm{C}$ & $5.4 \mathrm{c}$ & $1005.3 \mathrm{ab}$ & $1216.0 \mathrm{a}$ & $0.61 \mathrm{a}$ \\
\hline & & $4^{\text {th day }}$ & $3.8 \mathrm{abc}$ & 18.30 & $2428 \mathrm{a}$ & $71.3 \mathrm{bc}$ & $17311.6 \mathrm{a}$ & $8.7 \mathrm{a}$ & $10900 \mathrm{a}$ & $1354.8 \mathrm{a}$ & $0.68 \mathrm{a}$ \\
\hline & Sandy & Every day & $3.8 \mathrm{abc}$ & 8.50 & $38.0 \mathrm{f}$ & 53.80 & $2044.4 \mathrm{e}$ & $1.0 \mathrm{e}$ & $446.0 \mathrm{C}$ & $496.3 \mathrm{C}$ & $0.25 \mathrm{c}$ \\
\hline & loam & $2^{\text {nd }} d g y$ & 3.5 abod & $5.3 \mathrm{e}$ & $30.3 \%$ & $40.0 \mathrm{e}$ & $1212.8 \mathrm{e}$ & $0.6 \mathrm{e}$ & $4523 \mathrm{C}$ & $491.3 \mathrm{C}$ & $0.25 \mathrm{c}$ \\
\hline & & $3^{\text {ed }}$ day & $4.3 \mathrm{ab}$ & $23.5 \mathrm{a}$ & $1150 \mathrm{e}$ & 62.3 or & 7164.50 & 3.60 & 18400 & $326.8 \mathrm{c}$ & $0.16 \mathrm{c}$ \\
\hline & & $4^{\text {th }}$ day & 3.3000 & $14.8 \mathrm{C}$ & 148.3 of & $64.8 \mathrm{c}$ & $9602.4 \mathrm{C}$ & $48 \mathrm{c}$ & 786.5 b & $952.8 \mathrm{~b}$ & $0.48 \mathrm{D}$ \\
\hline \multirow{8}{*}{$\begin{array}{l}\text { squash } \\
\text { Ta barak F1 }\end{array}$} & Loamy & Every day & $12.8 \mathrm{a}$ & $130 \mathrm{c}$ & 630.30 & 42.3 cose & $26661.7 \mathrm{C}$ & $13.3 \mathrm{c}$ & $18478 \mathrm{a}$ & $2503.8 \mathrm{a}$ & $1.25 \mathrm{a}$ \\
\hline & sand & $2^{\text {nd }} \mathrm{day}$ & 3.80 & $29.5 \mathrm{ab}$ & 68600 & $65.0 \mathrm{~b}$ & $44590.5 \mathrm{~b}$ & $22.3 \mathrm{~b}$ & 387.0 de & 1105.30 & 0.550 \\
\hline & & $3^{\text {nd }}$ day & 1.00 & $30.8 \mathrm{ab}$ & $243.8 \mathrm{f}$ & $36.3 \mathrm{e}$ & 8849.9 e & $4.4 \mathrm{e}$ & $1125.3 \mathrm{bc}$ & 1400.800 & 0.7000 \\
\hline & & $4^{\text {th }}$ day & 4.30 & $32.8 \mathrm{a}$ & $346.3 \mathrm{e}$ & $52.3 \mathrm{c}$ & 18111.50 & 9.10 & $1155.3 \mathrm{bc}$ & $1538.5 \mathrm{C}$ & $0.77 \mathrm{c}$ \\
\hline & Sandy & Every day & $2.8 \mathrm{bc}$ & $15.0 \mathrm{c}$ & $562.5 \mathrm{C}$ & $47.8 \mathrm{~cd}$ & $26859.4 \mathrm{C}$ & $13.4 \mathrm{C}$ & 607.30 & 1187.50 & 0.590 \\
\hline & loam & $2^{\text {nd day }}$ & 1.5 or & $25.0 \mathrm{~b}$ & $9380 \mathrm{a}$ & $79.3 \mathrm{a}$ & $74383.4 \mathrm{a}$ & $372 \mathrm{a}$ & $903.8 \mathrm{C}$ & $1288.3 \mathrm{~b}$ & $0.93 b$ \\
\hline & & $3^{\text {rd }}$ day & 0.80 & $12.0 \mathrm{c}$ & 426.30 & $40.0 \mathrm{de}$ & 17052.50 & 8.50 & $1221.0 \mathrm{~b}$ & $1660.0 \mathrm{bc}$ & 0.8300 \\
\hline & & $4^{\text {th }}$ day & 0.0 & 3.80 & $111.8 \mathrm{~g}$ & $31.0 \mathrm{e}$ & $3465.8 \mathrm{e}$ & $1.7 \mathrm{e}$ & $2930 \mathrm{e}$ & $408.5 \mathrm{e}$ & $0.20 \mathrm{e}$ \\
\hline \multirow{8}{*}{$\begin{array}{l}\text { Cowpea } \\
\text { Qaha } 1\end{array}$} & Loamy & Every day & 0.0 & $17.8 \mathrm{ab}$ & $333.3 \mathrm{~b}$ & $105.8 \mathrm{a}$ & 35263.1 a & $17.6 \mathrm{a}$ & $50240 \mathrm{a}$ & $5375.0 \mathrm{a}$ & $2.69 \mathrm{a}$ \\
\hline & sand & $2^{\text {nd }} \mathrm{day}$ & 0.0 & $12.0 \mathrm{c}$ & $380.3 \mathrm{a}$ & 1000 ab & $36000.5 \mathrm{a}$ & $190 \mathrm{a}$ & $2340.5 \mathrm{C}$ & $2732.8 \mathrm{C}$ & $1.37 \mathrm{C}$ \\
\hline & & $3^{\text {rd }}$ day & 0.0 & $14.3 \mathrm{bc}$ & 3460 D & $105.8 \mathrm{a}$ & $36605.8 \mathrm{a}$ & $18.3 \mathrm{a}$ & $1792.3 \mathrm{c}$ & 2152.50 & 1.080 \\
\hline & & $4^{\text {th }}$ day & 0.0 & $12.5 \mathrm{c}$ & $2200 \mathrm{c}$ & $45.3 \mathrm{e}$ & $9966.8 \mathrm{C}$ & $5.0 \mathrm{c}$ & 1127.30 & $1359.8 \mathrm{e}$ & $0.68 \mathrm{e}$ \\
\hline & Sandy & Every day & 8.30 & $15.5 \mathrm{bc}$ & 379.5 a & $86.0 \mathrm{bC}$ & $32637.0 \mathrm{ab}$ & $16.3 \mathrm{ab}$ & $3852.0 \mathrm{~b}$ & $4255.3 \mathrm{~b}$ & $2.13 \mathrm{~b}$ \\
\hline & loam & $2^{\text {nd }} \mathrm{day}$ & $21.8 \mathrm{a}$ & $20.0 \mathrm{a}$ & 359.3 ab & 77.3 or & 2777390 & 1390 & $5305.8 \mathrm{a}$ & $5707.8 \mathrm{a}$ & $2.85 \mathrm{a}$ \\
\hline & & $3^{\text {nd }}$ day & 6.30 & $17.8 \mathrm{ab}$ & 14600 & 70.00 & $102200 \mathrm{c}$ & $5.1 \mathrm{C}$ & 900.50 & $1070.5 \mathrm{ef}$ & 0.54 ef \\
\hline & & $4^{\text {th }}$ day & 0.0 & 780 & $76.0 \mathrm{e}$ & 67.30 & $51148 \mathrm{C}$ & $26 \mathrm{c}$ & 536.00 & $619.8 \mathrm{f}$ & $0.31 \mathrm{f}$ \\
\hline
\end{tabular}

In each column, values of a cop cu rivars followed by the same letter(s) are not signilicantly of ferent $(\mathrm{P}=0.05)$. 
Table (5): Growth of eggplant, squash and cowpea infected with Rotylenchulus reniform is and grown in two different soil types as affected by the duration of irrigation.

\begin{tabular}{|c|c|c|c|c|c|c|c|c|c|}
\hline \multirow{2}{*}{ Grop } & \multirow{2}{*}{$\begin{array}{l}\text { solll } \\
\text { type }\end{array}$} & \multirow{2}{*}{$\begin{array}{l}\text { Irrigation } \\
\text { duration }\end{array}$} & \multicolumn{3}{|c|}{ Shoot } & \multicolumn{2}{|c|}{ Root } & \multirow{2}{*}{$\begin{array}{c}\begin{array}{c}\text { Total } \\
\text { langth }\end{array} \\
(\mathrm{cm})\end{array}$} & \multirow{2}{*}{$\begin{array}{c}\begin{array}{c}\text { Total fres } h \\
\text { wel ght }\end{array} \\
\text { (gm) }\end{array}$} \\
\hline & & & le ngth $(\mathrm{cm})$ & fres h wel ght (gm) & dry w elght (gm) & length(cm) & fres $\mathrm{h}$ wel gint (gm) & & \\
\hline \multirow{10}{*}{$\begin{array}{l}\text { Eggplant } \\
\text { Gloya na F1 }\end{array}$} & \multirow{5}{*}{$\begin{array}{l}\text { Loamy } \\
\text { sand }\end{array}$} & Every day & $24.05 \mathrm{~b}$ & $3.00 \mathrm{~b}$ & $0.52 \mathrm{~b}$ & $23.00 \mathrm{a}$ & $1.34 \mathrm{~b}$ & $47.05 \mathrm{~b}$ & $4.35 \mathrm{~b}$ \\
\hline & & $2^{\text {nd }} \mathrm{day}$ & 20.10 od & $1.36 \mathrm{de}$ & 0.200 & $16.33 \mathrm{C}$ & $0.55 \mathrm{c}$ & 36.43 d & 1910 \\
\hline & & $3^{\text {dd }} \mathrm{day}$ & $21.85 \mathrm{DC}$ & 1.66 od & $0.29 \mathrm{c}$ & $25.33 \mathrm{a}$ & $1.40 \mathrm{D}$ & $47.18 \mathrm{D}$ & $3.05 \mathrm{c}$ \\
\hline & & $4^{\text {th }}$ day & $17.50 \mathrm{e}$ & $1.24 \mathrm{e}$ & 0.17 de & $12.25 \mathrm{de}$ & $0.42 \mathrm{C}$ & 29.75 f9 & 1.65 def \\
\hline & & Heaithy & $26.43 \mathrm{a}$ & $4.60 \mathrm{a}$ & $0.95 \mathrm{a}$ & $26.00 \mathrm{a}$ & $2.90 \mathrm{a}$ & $52.48 \mathrm{a}$ & $7.50 \mathrm{a}$ \\
\hline & \multirow{5}{*}{$\begin{array}{l}\text { Sandy } \\
\text { Ioam }\end{array}$} & Every day & $16.73 \mathrm{e}$ & $0.63 \mathrm{~g}$ & $0.08 t$ & $10.33 \mathrm{e}$ & $0.44 \mathrm{c}$ & $27.05 \mathrm{~g}$ & $1.07 \mathrm{~g}$ \\
\hline & & $2^{24} d a y$ & $16.93 \mathrm{e}$ & $0.76 \% 9$ & $0.13 \mathrm{ef}$ & 14.50 or & $0.50 \mathrm{c}$ & $31.43 \mathrm{ef}$ & $1.25 \%$ \\
\hline & & $3^{\text {rd }} d a y$ & $17.03 \mathrm{e}$ & $0.83 \mathrm{fg}$ & 0.11 ef & 13.25 ode & $0.64 \mathrm{c}$ & 30.28 f & 1.47 etg \\
\hline & & $4^{\text {th }}$ day & 18.50 de & $1.11 \mathrm{ef}$ & 0.15 de & $16.00 \mathrm{c}$ & $0.63 \mathrm{c}$ & 34.50 de & $1.73 \mathrm{de}$ \\
\hline & & Hea ithy & 20.5000 & $1.80 \mathrm{c}$ & 0.550 & $20.00 \mathrm{~b}$ & $1.40 \mathrm{~b}$ & $40.50 \mathrm{c}$ & $3.20 \mathrm{c}$ \\
\hline \multirow{10}{*}{$\begin{array}{l}\text { Squash } \\
\text { Ta barak F1 }\end{array}$} & \multirow{5}{*}{$\begin{array}{l}\text { Loamy } \\
\text { sand }\end{array}$} & Every day & $32.75 \mathrm{a}$ & $5.47 \mathrm{~b}$ & $0.71 \mathrm{c}$ & $2200 \mathrm{~b}$ & $1.23 \mathrm{~b}$ & $54.75 \mathrm{ab}$ & $6.70 \mathrm{~b}$ \\
\hline & & $2^{2 d} d a y$ & $28.00 \mathrm{bC}$ & 3.98 od & 0.62 od & $18.75 \mathrm{C}$ & $0.93 \mathrm{bc}$ & $46.75 \mathrm{C}$ & $490 \mathrm{C}$ \\
\hline & & $3^{\text {nd }} \mathrm{day}$ & $25.00 \mathrm{C}$ & $1.63 \%$ & 0.32 ef & $19.25 \mathrm{C}$ & $0.80 \mathrm{dc}$ & 44.25 or & $2.43 \mathrm{e}$ \\
\hline & & $4^{\text {th }}$ day & $25.25 \mathrm{C}$ & $1.10^{f}$ & $0.21 \mathrm{f}$ & 17.78 od & $0.67 \mathrm{bc}$ & 43.03 or & $1.77 \mathrm{e}$ \\
\hline & & Hea ithy & $33.03 \mathrm{a}$ & $7.68 \mathrm{a}$ & $120 \mathrm{a}$ & $24.00 \mathrm{ab}$ & $2.45 \mathrm{a}$ & $57.03 \mathrm{a}$ & $10.12 \mathrm{a}$ \\
\hline & \multirow{5}{*}{$\begin{array}{l}\text { Sandy } \\
\text { Ioam }\end{array}$} & Every day & $28.68 \mathrm{bc}$ & $4.77 \mathrm{bc}$ & $0.67 \mathrm{c}$ & $24.18 \mathrm{ab}$ & $198 \mathrm{a}$ & $52.85 \mathrm{D}$ & $6.74 \mathrm{~b}$ \\
\hline & & $2^{2 d} d a y$ & $28.45 \mathrm{DC}$ & 3.70 os & 0.46 de & $23.63 \mathrm{~b}$ & 1.250 & $52.08 \mathrm{D}$ & $4.95 \mathrm{c}$ \\
\hline & & $3^{r d} \mathrm{day}$ & $26.25 \mathrm{c}$ & 3.15 de & $0.41 \mathrm{e}$ & 17.25 od & $0.83 \mathrm{bc}$ & 43.50 or & 3.98 od \\
\hline & & $4^{\text {th }}$ day & $26.00 \mathrm{C}$ & $1.97 \mathrm{ef}$ & 0.29 ef & 15.67 d & $0.60 \mathrm{c}$ & $41.67 \mathrm{~d}$ & $2.57 \mathrm{de}$ \\
\hline & & Hea ithy & $31.03 \mathrm{ab}$ & $7.23 \mathrm{a}$ & 1.000 & $26.48 \mathrm{a}$ & $2.38 \mathrm{a}$ & $57.50 \mathrm{a}$ & $9.60 \mathrm{a}$ \\
\hline
\end{tabular}


Table (5). Continued

\begin{tabular}{|c|c|c|c|c|c|c|c|c|c|}
\hline \multirow{2}{*}{ Crop } & \multirow{2}{*}{$\begin{array}{l}\text { soll } \\
\text { ty pe }\end{array}$} & \multirow{2}{*}{$\begin{array}{l}\text { Irrigation } \\
\text { duration }\end{array}$} & \multicolumn{3}{|c|}{ shoot } & \multicolumn{2}{|r|}{ Root } & \multirow{2}{*}{$\begin{array}{c}\begin{array}{c}\text { Total } \\
\text { longth }\end{array} \\
\text { (cm) }\end{array}$} & \multirow{2}{*}{$\frac{\text { Total tresh w el ght }}{(g \mathrm{~m})}$} \\
\hline & & & length (cm) & fresh weight (gm) & dry w elght (gm) & length $[\mathrm{cm})$ & fres h wel ght (gm) & & \\
\hline \multirow{10}{*}{$\begin{array}{l}\text { Cowpea } \\
\text { Qaha } 1\end{array}$} & \multirow{5}{*}{$\begin{array}{l}\text { Loamy } \\
\text { sand }\end{array}$} & Every day & $30.25 \mathrm{~b}$ & $9.53 \mathrm{~b}$ & $1.20 \mathrm{ab}$ & $26.75 \mathrm{a}$ & $3.09 \mathrm{~b}$ & $57.00 \mathrm{a}$ & $1261 \mathrm{~b}$ \\
\hline & & $2^{\text {dd }}$ day & $27.25 \mathrm{~b}$ & $7.60 \mathrm{c}$ & 0.87 of & $22.38 \mathrm{bc}$ & $3.80 \mathrm{a}$ & $50.23 \mathrm{DCC}$ & $11.40 \mathrm{C}$ \\
\hline & & $3^{\text {rd }} \mathrm{day}$ & $30.30 \mathrm{~b}$ & 6.430 & $1.07 \mathrm{bc}$ & $21.25 \mathrm{c}$ & $2.44 \mathrm{C}$ & $51.55 \mathrm{~b}$ & 8.870 \\
\hline & & $4^{\text {th }}$ day & $25.03 \mathrm{C}$ & $4.80 \mathrm{e}$ & 0.77 de & $23.25 \mathrm{bc}$ & 1.300 & $48.28 \mathrm{DCC}$ & $6.10 \mathrm{ef}$ \\
\hline & & Healthy & $33.00 \mathrm{a}$ & $11.20 \mathrm{a}$ & $1.40 \mathrm{a}$ & $27.00 \mathrm{a}$ & $4.23 \mathrm{a}$ & $60.00 \mathrm{a}$ & $15.43 \mathrm{a}$ \\
\hline & \multirow{5}{*}{$\begin{array}{l}\text { Sandy } \\
\text { loam }\end{array}$} & Every day & $20.35 \mathrm{e}$ & $4.55 \mathrm{e}$ & $0.63 \mathrm{ef}$ & $22.50 \mathrm{bc}$ & $2.20 \mathrm{c}$ & 42.850 & $6.75 \mathrm{e}$ \\
\hline & & $2^{\text {nd day }}$ & $20.88 \mathrm{de}$ & $4.20 \mathrm{e}$ & 0.47 g & $21.63 \mathrm{bc}$ & 1.110 & $42.50 \mathrm{~d}$ & $5.31 \mathrm{f}$ \\
\hline & & $3^{\text {nd }}$ day & $19.83 \mathrm{e}$ & $400 \mathrm{e}$ & $0.50 \mathrm{fg}$ & $22.00 \mathrm{bc}$ & $3.05 \mathrm{~b}$ & 41.830 & $7.06 \mathrm{e}$ \\
\hline & & $4^{\text {th }}$ day & $16.43 i$ & $1.53 \mathrm{f}$ & $0.30 \mathrm{~g}$ & 24.75 ab & $195 \mathrm{c}$ & $41.18 \mathrm{~d}$ & $3.48 \mathrm{~g}$ \\
\hline & & Healthy & 23.0000 & 6.8000 & $1.10 \mathrm{~b}$ & $2400 a b c$ & $3.93 \mathrm{a}$ & $47.00 \mathrm{C}$ & $10.73 \mathrm{c}$ \\
\hline
\end{tabular}

In each column, values of a crop cu fivars fo lowed by the same letter( $(8)$ are not signincantly offerent ( $P=0.05)$. 


\section{Discussion}

Results of the influence of water stress on the reproduction of the root-knot and the reniform nematodes indicated that such influence depends to great extent on soil type, nematode species and the cultivated crop. As for the root-knot nematode, Meloidogyne incognita, the present results showed that the highest nematode counts were observed on roots of eggplant grown in loamy sand soil and irrigated at 3 days intervals (at the fourth day). However, on squash, the highest nematode counts were recorded on plants grown in sandy loam soil and irrigated every day. On cowpea, different behavior was observed whereas the highest significant nematode counts were recorded on roots of plants grown in sandy loam soil and irrigated every four days followed by those grown in loamy sand and irrigated at the same interval. Apparently, no significant differences could be observed within and between different irrigation intervals in both types of soil.

The highest numbers of egg-masses/root, eggs/g soil, total number of eggs, final population as well as the rate of reproduction of the reniform nematode, Rotylenchulus reniformis on eggplant roots were accomplished in loamy sand soil irrigated every four days. Irrespective of intervals of irrigation, nematode counts in the loamy sand soil were significantly higher than those in the sandy loam soil. Generally, increasing soil moisture (daily irrigation) was not in favor to nematode reproduction, however, prolonging the period of irrigation enhanced the reniform nematode reproduction. Conversely, increasing soil moisture (daily irrigation or irrigation every other day) was found to be in favor to nematode reproduction on squash and cowpea especially in loamy sand soil. The most significant highest final population and rate of reproduction on squash was recorded in loamy sand soil irrigated every day and on cowpea in sandy loam soil irrigated every other day.

Regarding growth response to the infection of the root-knot and the reniform nematodes and the stress of soil moisture, present data illustrated that prolonging irrigation period (decreasing soil moisture) significantly reduced the growth of the infected plants. Such effect was pronounced with the longest period between irrigation ( 3 days) and in loamy sand soil.

The present results are supported by the opinion of Norton, 1978 when he said that, soil moisture varies considerably as a result of many biological and physical actions. In the broad sense, some of the most obvious factors regulating soil moisture are intensity and frequency of irrigation, rainfall, the drainage pattern, and the precipitation/evaporation ratio. Other factors governing the amount of moisture in nematode's environment include the amount of available soil moisture for the particular soil type, the stage of plant maturity, the length of growing seasons, the water requirements of individual plants or crops, and elements relating to individual characteristics of leaves and roots. The relative capacity of moisture is so dependent upon the soil texture and other factors that determine the soil phase 
that they cannot be separated. The coarser the soil, the larger the pores, the smaller the total soil surface area, the less water retained. Clays, which are composed of extremely small particles, have an enormous surface area, small pores and thus a great water retaining ability.

Movement, development and survival of nematodes in soil are regulated by the interaction between soil porosity and water potential. Depending on pore size and hydrology, a nematode may reside in a pore that is filled with water or in a film of water adhering to soil particle or root surface. Large, water-filled soil pores require nematodes to swim, an inefficient form of locomotion for most species. Reduced aeration in saturated soil is often detrimental to nematodes (Sotomayor et al., 1999; Soriano et al., 2000), although it can induce quiescence to extend longevity in some species (Van Gundy et al., 1967).

As water drains or evaporates become increasingly constrained within a film of water and locomotion is more efficient due to the resistance to nematode thrusting provided by the surface tension of the water film. This may illustrate the high rates of reproduction of the reniform nematode wherever the frequency of irrigation decreased. Egg hatch is the most important biological process influenced by soil moisture. Van Gundy (1958) speculated that the interval between irrigations of citrus infected with Tylenculus semipenetrans is important in the life cycle of the nematode because free water necessary to flush the juveniles from the eggmasses.

\section{References}

Bakonyi, G.; Nagy, P.; Kovacs-Lang, E.; Kovacs, E.; Barabas, S.; Repasi, V. and Seres, A. (2007). Soil nematode community structure as affected by temperature and moisture in temperate semiarid shrubland. Applied Soil Ecology (2007): 31-40.

Couch, H. B. and Bloom, J. R. (1960). Influence of soil moisture stresses on the development of the root-knot nematode. Phytopathology 50:319-321.

Daulton, R. A. C. and Nusbaum, C. J. (1962). The effect of soil moisture and humidity on the root-knot nematode Meloidogyne javanica. Nematologica, 8(2)157-168.

Kable, P.F. and Mai, W.F. (1968). Infeluence of soil moisture on Pratylenchus penetrans. Nematologica, 14: 101-122.

Khanizadeh, h; Belair, G and Lareau, M.j. (1994). Relative susceptibility of five strawberry cultivars to Meloidogyne hapla under three soil water deficit levels. Phytoprotection, 75( 3): 133-137. 
Norton, D.C. (1978). Ecology of Plant Parasitic Nematodes. A. Wiley- Interscience publication, 2nd Ed. New York, USA, pp 268.

Rebois, R. V. (1973). Effect of soil water in infectivity and development of Rotylenchulus reniformis on soybean, Glycine max. Journal of Nematology, 5:246-249.

Soriano, I. R. S.; Port, J. C. and Matias, D. M. (2000). Expression of tolerance for Meloidogyne graminicola in rice cultivars as affected by soil type and flooding. Journal of Nematology.(32):309-317.

Sotomayer, D., Allen, L. H.; Jr, Chen, Z.; Dickson, D. W. And Hewlett. T. (1999). Anaerobic soil management practices and solarization for nematode. Nematologica, (36): 448-456.

SPSS (2003). Statistical package for the social science Incorporation, Chicago, SPSS base 12.0 application guide. Chicago: SPSS, X1 426 pp.

Van Gundy, S. D. (1958). The life history of the citrus nematode. Tylenchulus semipenetrance Cobb. Nematologica (3): 283-294.

Van Gundy, S. D.; Bird, A. F. And Wallace, H. R. (1967). Aging and starwation in larva of Meloidogyne javanica and Tylenchulus semipenetrans. Phytopathology (57): 559-571. 


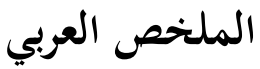

تأثير مواعيد الري على إصابة وتكاثر كل من نيماتودا تعقد الجذور والنيماتودا الكلوية لبعض محاصيل الخضر النامية في نوعين مختلفين من التربة.

أحمد عبد السلام فرحات ، السيد أبو المعاطي السيد، شيماء فتحي دياب قسم الحيوان والنيماتولوجيا الزراعية - كلية الزراعة - جامعة القاهرة

تم في هذا البحث دراسة تأثير التغير في رطوبة التربة والناتج عن التغير في مواعيد الري على التي

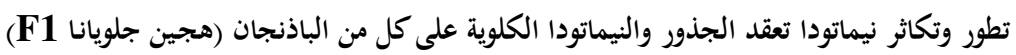

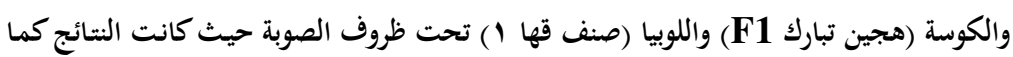

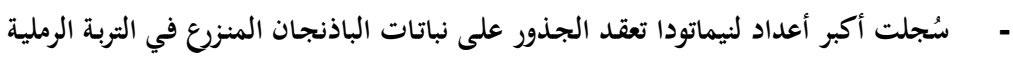

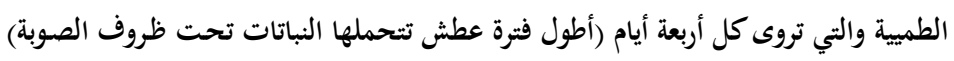

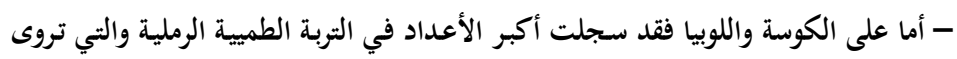
يوميًا أو كل أربعة أيام على التوالي.

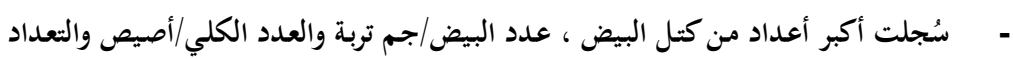

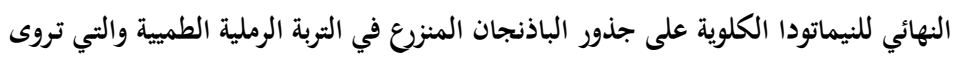

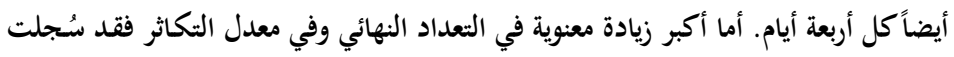
في نفس نوع التربة على الكوسة واللوبيا والتي تروى يومياً أو يوما بعد يوم ، على التواد التوالي. 